\title{
Right Atrial Thrombosis after Upgrading to a Biventricular Pacing/Defibrillation System
}

\author{
Satoshi Kurisu, Ichiro Inoue and Takuji Kawagoe
}

\begin{abstract}
A 56-year-old man under right ventricular pacing for atrial fibrillation and bradycardia had congestive heart failure. He received a cardiac resynchronization pacemaker with a defibrillator. Four months later, follow-up transthoracic echocardiography showed a right atrial mass although he had no symptom. Transesophageal echocardiography showed a large immobile round-shaped mass on the defibrillation lead, which was attached to the free wall of the right atrium. One month after the initiation of anticoagulant therapy, the mass disappeared, suggesting that it was thrombotic. During the 5 month follow-up, he remained in good condition without the recurrence of right atrial thrombosis.
\end{abstract}

Key words: pacemaker, thrombosis

(Inter Med 48: 2101-2104, 2009)

(DOI: 10.2169/internalmedicine.48.2453)

\section{Introduction}

The number of patients who receive pacemaker or implantable cardioverter defibrillator is steadily increasing. On the other hand, pacing lead is known to be a predisposing factor of thrombosis (1-3). This report describes a case of right atrial thrombosis after upgrading to a biventricular pacing/defibrillation system that is diagnosed as an incidental echocardiographic finding.

\section{Case Report}

A 56-year-old man, who had been treated with ticlopidine (200 mg/day) and ventricular pacing for atrial fibrillation and bradycardia, was admitted to our hospital because of orthopnea. Chest X-ray showed mild pulmonary congestion, and electrocardiogram (ECG) showed atrial fibrillation and ventricular pacing with a QRS duration of $180 \mathrm{msec}$. Transthoracic echocardiography showed left ventricular diameter of $52 \mathrm{~mm}$, left atrial diameter of $55 \mathrm{~mm}$ and ejection fraction of $45 \%$ (Table 1). Mild mitral regurgitation and intraventricular dyssynchrony were found, but left ventricular hypertrophy was not found. Coronary angiography showed no coronary artery disease. Telemetry monitoring docu- mented non-sustained ventricular tachycardia. According to these results, he was diagnosed with congestive heart failure in part associated with intraventricular dyssynchrony due to ventricular pacing. He was treated with intravenous furosemide and carperitide. After the treatment, he received a cardiac resynchronization pacemaker with a defibrillator, which resulted in a decreased QRS duration of $120 \mathrm{msec}$ (Fig. 1). The defibrillator lead was located at the right ventricular apex, and the left ventricular lead was located at the posterolateral vein. After the procedure, he was followed up at his local clinic with imidapril $(2.5 \mathrm{mg} / \mathrm{day})$ and ticlopidine (200 mg/day).

Four months later, follow-up transthoracic echocardiography showed a right atrial mass $(31 \times 26 \mathrm{~mm})$ on subcostal view (Fig. 2A, arrow) although he had no symptom. Transesophageal echocardiography showed smoking echo contrast in both right and left atria and reduced peak velocity of 24 $\mathrm{cm} / \mathrm{s}$ in the left atrial appendage. No mass was found in the left atrial appendage (Fig. 2B). However, a large immobile round-shaped mass was found on the defibrillation lead (Fig. 2C, 2D, arrows). It was attached to the free wall of the right atrium. Computed tomography also showed a right atrial mass (Fig. 3, arrow), but did not show any findings of pulmonary embolism. Laboratory examination revealed the following findings: leukocyte count, 4,600/mm; hemoglobin, 

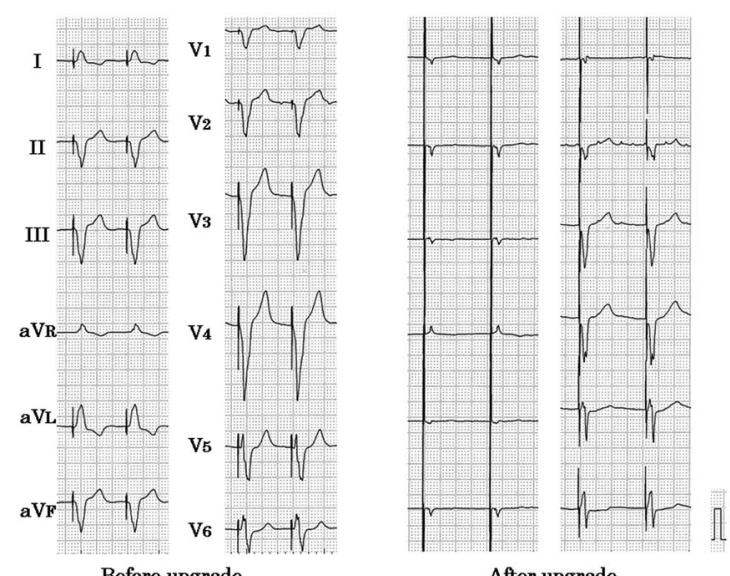

Figure 1. Electrocardiograms before and after upgrading to a biventricular pacing/defibrillation system. QRS duration decreased from $180 \mathrm{msec}$ to $120 \mathrm{msec}$.
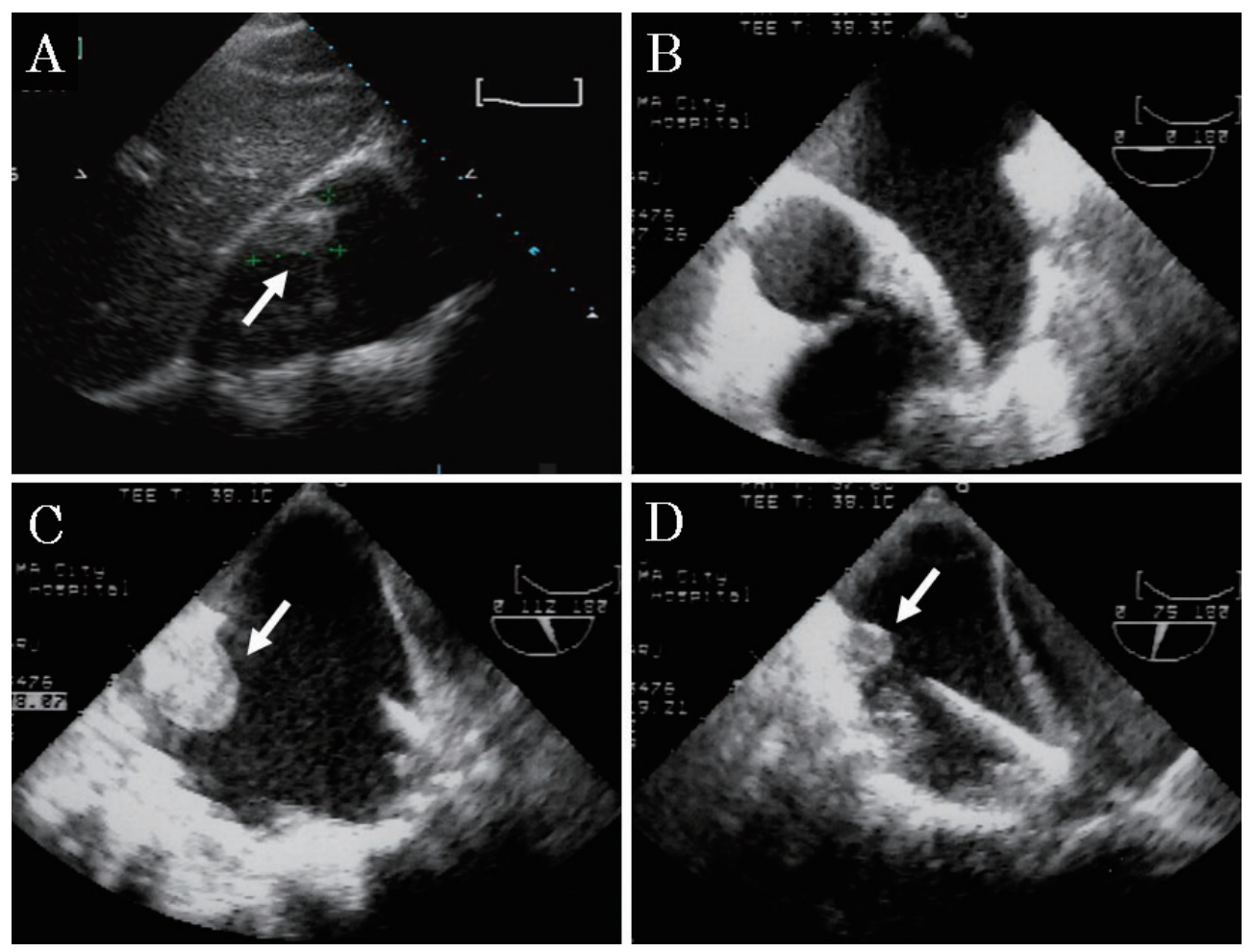

Figure 2. Follow-up transthoracic echocardiography showed a right atrial mass $(31 \times 26 \mathrm{~mm})$ on subcostal view (A, arrow). Transesophageal echocardiography showed no mass in the left atrial appendage $(B)$, but did reveal a large immobile round-shaped mass on the defibrillation lead, which was attached to the free wall of the right atrium (C and D, arrows).

$11.9 \mathrm{~g} / \mathrm{dL}$; platelet count, $21.2 \times 10^{4} / \mathrm{mm} ; \mathrm{BUN}, 6 \mathrm{mg} / \mathrm{dL}$; creatinine, $0.62 \mathrm{mg} / \mathrm{dL}$; creatine kinase, $84 \mathrm{IU} / \mathrm{L}$; sodium, $133.8 \mathrm{mEq} / \mathrm{L}$ and potassium, $3.1 \mathrm{mEq} / \mathrm{L}$. Fibrin degradation product was normal $(3.8 \mu \mathrm{g} / \mathrm{mL})$ and D-dimer was increased $(2.3 \mu \mathrm{g} / \mathrm{mL})$. Tumor makers including CEA, CA19-9 and SCC were normal. Antinuclear antibody and blood culture were negative.

Because the mass, which was possibly thrombotic in nature, was immobile, anticoagulant therapy was initiated. The prothrombin time (international normalized ratio) was meticulously kept between 2.0 and 3.0 by using warfarin. One month after the initiation, the mass disappeared, suggesting that it was thrombotic. Anticoagulant therapy was continued with imidapril. During the 5 month follow-up, he remained in good condition without the recurrence of right atrial thrombosis. 


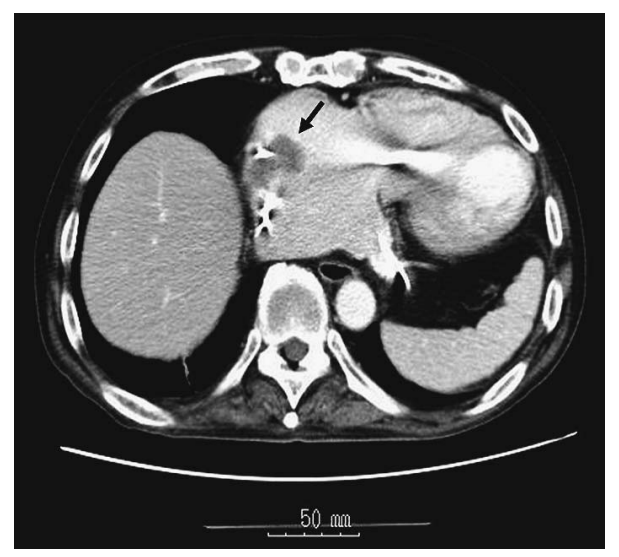

Figure 3. Computed tomography showed a right atrial mass on the defibrillation lead, which was attached to the free wall of the right atrium.

Table 1. Time Course of Echocardiographic Findings

\begin{tabular}{lccc}
\hline & Before upgrade & 4 months later & 7 months later \\
\hline Left ventricular end-diastolic diameter $(\mathrm{mm})$ & 52 & 46 & 49 \\
Left ventricular end-systolic diameter $(\mathrm{mm})$ & 37 & 31 & 32 \\
Ejection fraction (\%) & 45 & 61 & 65 \\
Left atrial diameter (mm) & 55 & 56 & 54 \\
Grade of mitral regurgitation & $\mathrm{I}$ & $\mathrm{I}$ & $\mathrm{I}$ \\
Grade of tricuspid regurgitation & trivial & trivial & trivial \\
Right atrial mass & Absent & Present & Absent \\
\hline
\end{tabular}

\section{Discussion}

In this report, we presented a case of right atrial thrombosis which occurred after upgrading to a biventricular pacing/ defibrillation system. The development of device therapy has improved quality of life, functional status and exercise capacity in patients with heart failure and bradycardia $(4,5)$. On the other hand, a recent study demonstrated that dual chamber rate response pacing worsened the combined end point of mortality and hospitalization for heart failure compared with ventricular backup pacing (6). A possible mechanism for the maladaptive effects was altered ventricular activation from right ventricular pacing. In the present case, ventricular pacing was essential for bradycardia, and upgrading to a biventricular pacing was performed for the treatment of heart failure.

Several etiologies of right atrial mass such as thrombus, infectious vegetation and malignant tumor were considered. Our patient did not have clinical findings of endocarditis or malignancy. Also, the mass developed during a period of 4 months in the right atrium involving the slowed blood flow, suggesting the diagnosis of thrombus.

Previous studies have reported that the incidence of pacing lead-induced venous thrombosis varies from $0.6 \%$ to $3.5 \% \%(1,2)$. However, right atrial thrombosis attached to a pacing lead is uncommon. Tugcu et al recently reported a case of right atrial pacemaker lead thrombosis (7). The thrombus $(32 \times 11 \mathrm{~mm})$ was smaller than that of our case, but it was mobile. Because the thrombus was partially prolapsing into the right ventricle during diastole and causing tricuspid inflow obstruction, it was surgically removed. In the present case, the thrombus was immobile, and was attached to the free wall of the right atrium. Because it had no hemodynamic significance, anticoagulant therapy was considered. The type of treatment should be determined according to the size, morbidity, location and duration of right atrial thrombus.

In the present case, several possible mechanisms were postulated in the pathogenesis of right atrial thrombosis. First, the patient had atrial fibrillation with smoking echo contrast in both atria. Slow evacuation of blood in the right atrium appeared to be associated with the thrombus formation. Second, 3 leads were involved in the right atrium after upgrading to biventricular pacing/defibrillation system. Single ventricular lead before upgrade has never resulted in right atrial thrombosis, suggesting that multiple leads might be associated with the thrombus formation. Third, defibrillation lead, which was thicker than the other leads, might cause the intraatrial endothelial injury with the subsequent thrombus formation.

The accumulation of risk factors increases thromboembolic event rates in patients with nonvalvular fibrillation, and CHADS2 score has recently been used for risk stratification (8). The present patient had only congestive heart failure among these risk factors. Because CHADS2 score 
was low, and antiplatelet therapy had a preventive effect of venous obstruction (9), our patient was treated with ticlopidine. The clinical course of our case demonstrated that warfarin had a preventive effect of right atrial thrombosis, but ticlopideine did not have such effect. It should be carefully examined on a case-by-case basis whether or not prophylactic anticoagulant therapy is necessary in patients undergoing device implantation.

\section{References}

1. Porath A, Avnun L, Hirsch M, Ovsyshcher I. Right atrial thrombus and recurrent pulmonary emboli secondary to permanent cardiac pacing: a case report and short review of the literature. Angiology 38: 627-630, 1987.

2. Barakat K, Robinson NM, Spurrell RAJ. Transvenous pacing leadinduced thrombosis: a series of cases with a review of the literature. Cardiology 93: 142-148, 2000.

3. Carda R, Almeria C, Lennie V, Serra V, Zamorano JL. What to do with an atrial thrombus? Eur J Echocardiogr 9: 204-205, 2008.

4. Duray GZ, Israel CW, Pajitnev D, Hohnloser SH. Upgrading to biventricular pacing/defibrillation systems in right ventricular paced congestive heart failure patients: prospective assessment of procedural parameters and response rate. Europace 10: 48-52, 2008.

5. Young JB, Abraham WT, Smith AL, et al. Combined cardiac resynchronization and implantable cardioversion defibrillation in ad- vanced chronic heart failure: the MIRACLE ICD Trial. JAMA 289: 2685-2694, 2003.

6. DAVID Trial Investigators. Dual-chamber pacing or ventricular backup pacing in patients with an implantable defibrillator. JAMA 288: 3115-3123, 2002.

7. Tugcu A, Yildirimturk O, Tayyareci $Y$, et al. Right atrial thrombosis pacemaker lead thrombosis causing tricuspid inflow obstruction. Pacing Clin Electrophysiol 32: 262-264, 2009.

8. Inoue H, Nozawa T, Hirai T, et al. Accumulation of risk factors increases risk of thromboembolic events in patients with nonvalvular atrial fibrillation. Circ J 70: 651-656, 2006.

9. Haghjoo M, Nikoo MH, Fazelifar AF, et al. Predictors of venous obstruction following pacemaker or implantable cardioverterdefibrillator implantation: a contrast venographic study on 100 patients admitted for generator change, lead revision, or device upgrade. Europace 9: 328-332, 2007.

(C) 2009 The Japanese Society of Internal Medicine http://www.naika.or.jp/imindex.html 